\section{Bosentan and oral anticoagulants in HIV patients: what we can learn of cases reported so far}

\author{
José Antonio Morales-Molina, ${ }^{1}$ \\ Juan Enrique Martínez-de la Plata, ${ }^{1}$ \\ Olivia Urquízar-Rodríguez, ${ }^{1}$ \\ María Angustias Molina-Arrebola ${ }^{2}$ \\ 1Pharmacy Department and \\ ${ }^{2}$ Haematology Department, Hospital de \\ Poniente, El Ejido, Almería, Spain
}

\section{Abstract}

Pulmonary arterial hypertension is an infrequent but nevertheless serious life-threatening severe complication of HIV infection. It can be treated with bosentan and oral anticoagulants. Bosentan could induce the acenocoumarol metabolism and it increases the INR values. Until now, no study of interaction between bosentan and oral anticoagulants in HIV patients has reported. So we present a case of this interaction between these drugs and we reviewed MEDLINE to identify all the papers published so far. In our case, several weeks after increasing dose of bosentan acenocoumarol dose had to be progressively increased to $70 \mathrm{mg} /$ week $(+33 \%)$ without obtaining an adequate INR level (2.0-3.0). Forty-nine days later, we achieved a therapeutic INR with $90 \mathrm{mg} /$ week of warfarin. The use of bosentan and oral anticoagulants together in these patients require a closer monitoring during first weeks of treatment, after increasing the bosentan dose and even during longer periods of time.

\section{Introduction}

Pulmonary arterial hypertension (PAH) is an infrequent but nevertheless serious lifethreatening severe complication of HIV infection. It is associated with an increase of pulmonary vascular resistance, pulmonary artery pressure, right-ventricular failure and death. Bosentan, a dual endothelin-1 receptor antagonist, is indicated in the treatment of PAH. It also improves exercise capacity, New York Heart Association (NYHA) functional class, quality of life as well as cardiopulmonary hemodynamics, cardiac geometry, and cardiac function in these patients. The combination of bosentan with antiretroviral therapy appears to be safe and to have no adverse impact on the control of HIV infection. ${ }^{1}$ However, PAH patients present a high risk of developing thromboembolism and thrombosis. Oral anticoagulants have been shown to improve survival in patients with pulmonary hypertension. ${ }^{2}$ Nowadays, HIV patients have a greater life expectancy compared to a few years ago. They can be treated with many drugs hepatically metabolized. Bosentan could induce both cytochrome P450 (CYP) 3A4 and CYP2C9 enzymes, which are also involved in acenocoumarol metabolism, and it is eliminated by biliary excretion. ${ }^{1}$ An alteration in the anticoagulant effect might increase the risk of haematological disturbances. Until now, the clinical data regarding the interaction between bosentan or ritonavir with oral anticoagulants (either warfarin or acenocoumarol) are limited., ${ }^{1,3-5}$ No study of interaction between bosentan and acenocoumarol has reported so far. So, we present a case of interaction between bosentan and oral anticoagulants in an HIV patient and we made a systematic review in MEDLINE to identify published papers in English to identify cases of interaction between bosentan and oral anticoagulants in HIV patients. The keywords used in the search were acenocoumarol, warfarin, bosentan, drug interactions, protease inhibitors, International Normalized Ratio and pulmonary arterial hypertension. Initially, we analyzed all cases found. The search covered the period from 1966 to 2011, the last search made was on 29 Jul 2011. The cases selected were those where there was interaction between the three drugs: bosentan, oral anticoagulants and protease inhibitors.

\section{Case Report}

In August 2009, a 48-year-old Caucasian male was diagnosed with PAH, associated with HIV infection. He also had a double aortic lesion with dilatation of the right cavities and he was being treated with acenocoumarol. The patient was an ex-smoker, ex-parenteral drug addict and had previously been diagnosed with chronic but stable hepatitis C (Child-Pugh Class A). On $24^{\text {th }}$ October 2009, he came to our Haematology Department for anticoagulation control. Over the previous year, his medication regimen had included lopinavir $200 \mathrm{mg} /$ ritonavir $50 \mathrm{mg} 2$ capsules twice daily and emtricitabine $200 \mathrm{mg} /$ tenofovir $300 \mathrm{mg}$ once daily. Over the previous month, he had taken acenocoumarol $56 \mathrm{mg} /$ week (achieving an INR values in the therapeutic range, 2.0-3.0). In the last week of September, he began treatment with bosentan $62.5 \mathrm{mg}$ twice daily. One month later, the bosentan dose was increased from $62.5 \mathrm{mg}$ to $125 \mathrm{mg}$ and two weeks after that, he presented an international normalized ratio (INR) of less than 2. The acenocoumarol dose was progressively increased up to $56 \mathrm{mg} /$ week
Correspondence: José Antonio Morales-Molina, $\mathrm{PhD}$, Pharmacy Department, Hospital de Poniente, 04700 - El Ejido, Almería, Spain.

Tel. +34.950.022.599 - Fax: +34.950 .022 .599$

E-mail: joseantonio.morales@ephpo.es

Key words: acenocoumarol, bosentan, drug interactions, warfarin, HIV patients.

Received for publication: 5 July 2011.

Accepted for publication: 16 September 2011.

This work is licensed under a Creative Commons Attribution NonCommercial 3.0 License (CC BYNC 3.0).

(C) Copyright J.A. Morales-Molina, et al. 2011

Licensee PAGEPress, Italy

Hematology Reports 2010; 3:e16

doi:10.4081/hr.2011.e16

to obtain an adequate INR level. In the last week of March 2010, the patient again presented with an INR of less than 2 (bosentan 125mg twice daily initiated on $8^{\text {th }}$ March 2010). The acenocoumarol dose was raised to $70 \mathrm{mg} /$ week $(+33 \%)$ but failed to produce an adequate INR level. On $21^{\text {th }}$ April 2010, in an attempt to avoid an interaction between bosentan and acenocoumarol, this was stopped. Then, warfarin 35 mg/week and bemiparin 7500 IU daily was initiated. Forty-nine days later, we achieved a therapeutic INR with $90 \mathrm{mg} /$ week $(+157 \%)$ of warfarin (Table 1). He denied using alcohol or tobacco, having changed his dietary intake, or either missing or taking extra doses of oral anticoagulants. Viral load remained undetectable ( $<50$ HIV RNA copies/mL) and lymphocytes CD4 + >300 cells $/ \mathrm{mm}^{3}$ during this period. Dose of lopinavir /ritonavir remained unchanged during bosentan and acenocoumarol treatment. After three months of treatment levels of INR remained between 2-3. The Horn Drug Interaction Probability Scale indicated a probable interaction between bosentan, acenocoumarol and lopinavir/ritonavir. $^{6}$

\section{Discussion}

Bosentan could increase the metabolization of both oral anticoagulants and develop a clinically-relevant decrease of oral anticoagulant concentrations. In our patient, it was necessary to significantly increase the dose of acenocoumarol and warfarin to try achieving a therapeutic INR. Spangler and Saxena reported that a patient treated with bosentan and warfarin needed a warfarin dose increase of $43 \%$ (75 mg/wk) to maintain the therapeutic INR. $^{3}$ In addition, Murphey and Hood reported a warfarin dose increase of $64 \%$ (45 mg/wk). ${ }^{4}$ 
Table 1. Anticoagulant history.

\begin{tabular}{|c|c|c|c|}
\hline Date & INR $^{*}$ & \multicolumn{2}{|c|}{ Anticoagulant dose, $\mathrm{mg} / \mathrm{wk}$} \\
\hline $10 / 24 / 09$ & 2.2 & $52.5(\mathrm{AC})$ & Bosentan dose $\uparrow$ from $62.5-125 \mathrm{mg}^{\circ}$ \\
\hline $11 / 10 / 09$ & 1.5 & 52.5 & $\mathrm{AC}$ dose $\uparrow$ to $53.5 \mathrm{mg} / \mathrm{wk}$ \\
\hline $11 / 19 / 09$ & 1.4 & 53.5 & $\mathrm{AC}$ dose $\uparrow$ to $56 \mathrm{mg} / \mathrm{wk}$ \\
\hline $11 / 26 / 09$ & 1.8 & 56 & - \\
\hline $12 / 04 / 09$ & 2.8 & 56 & - \\
\hline $2 / 15 / 10$ & 2.6 & 56 & - \\
\hline $3 / 23 / 10$ & 1.6 & 56 & $\begin{array}{l}\text { Bosentan } 125 \mathrm{mg} \text { BID initiated on } \\
\text { 3/08/10; AC dose } \uparrow \text { to } 63 \mathrm{mg} / \mathrm{wk}\end{array}$ \\
\hline $4 / 14 / 10$ & 1.7 & 63 & $\mathrm{AC}$ dose $\uparrow$ to $70 \mathrm{mg} / \mathrm{wk}$ \\
\hline $4 / 21 / 10$ & 1.7 & 70 & $\begin{array}{c}\text { AC was stopped. Warfarin } 35 \mathrm{mg} / \mathrm{wk} \\
\text { and subcutaneous bemiparin } 7500 \\
\text { IU/day was initiated }\end{array}$ \\
\hline $4 / 26 / 10$ & 1.2 & 35 & Warfarin dose $\uparrow$ to $42.5 \mathrm{mg} / \mathrm{wk}$ \\
\hline $5 / 06 / 10$ & 1.3 & 42.5 & Warfarin dose $\uparrow$ to $50 \mathrm{mg} / \mathrm{wk}$ \\
\hline $5 / 13 / 10$ & 1.3 & 50 & Warfarin dose $\uparrow$ to $57.5 \mathrm{mg} / \mathrm{wk}$. \\
\hline $5 / 20 / 10$ & 1.4 & 57.5 & Warfarin dose $\uparrow$ to $62.5 \mathrm{mg} / \mathrm{wk}$ \\
\hline $5 / 27 / 10$ & 1.4 & 62.5 & Warfarin dose $\uparrow$ to $70 \mathrm{mg} / \mathrm{wk}$ \\
\hline 6/03/10 & 1.5 & 70 & Warfarin dose $\uparrow$ to $90 \mathrm{mg} / \mathrm{wk}$ \\
\hline $6 / 09 / 10$ & 3 & 90 & Bemiparin was stopped \\
\hline $6 / 29$ & 2.5 & 90 & - \\
\hline $7 / 14$ & 2 & 90 & - \\
\hline $8 / 05 / 10$ & 2.3 & 90 & - \\
\hline
\end{tabular}

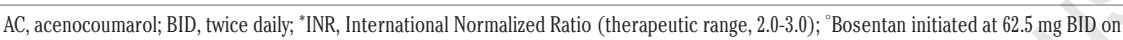
9/24/09.

In both cases, the interaction occurred in the first 10 days of treatment with bosentan while in our case it occurred on the $40^{\text {th }}$ day and he needed a higher dose (20-50\%) of warfarin to reach a therapeutic INR. ${ }^{2,3}$ In other reported cases, after increasing the bosentan dose our patient presented a delay in the response of more than 1 month. ${ }^{3}$ In other case, Llibre et al. reported a dramatic drop of INR after concomitant treatment with ritonavir and acenocoumarol. ${ }^{5}$ In our patient, this interaction was not shown. After a month of treatment with lopinavir/ritonavir and acenocoumarol, our patient presented a stable value of INR, viral load and CD4 + lymphocytes. The INR values only dropped drastically after receiving two months treatment of bosentan and acenocoumarol. He required very high doses of warfarin in order to achieve an INR within the therapeutic range. The dosage of $90 \mathrm{mg} /$ week could indicate a possible induction of warfarin metabolism. Dingemanse and van Giersbergen observed an increase in bosentan concentration up to 48-fold during the first 4 days of coadministration with lopinavir/ritonavir. ${ }^{7}$ In our case, lopinavir/ritonavir did not seem to raise bosentan level. However, bosentan could significantly compromise the metabolism of both oral anticoagulants. ${ }^{8}$

Several mechanisms might explain this interaction. Firstly, in patients with mild hepatic impairment (Child-Pugh class A), such as the case in our patient, an increase of the bosentan dose along with its active metabolite, Ro 48-5033, has been reported. ${ }^{9}$ In addition, Dingemanse and van Giersbergen reported that the exposure to bosentan in patients with PAH could present with low hepatic clearance. ${ }^{7}$ An increase in the bosentan dose could reduce the bile salt export pump level by up to $78 \%$, increasing its half-life. ${ }^{10}$ In our patient, the delay in the onset of symptoms could be related to a reduction in the hepatic uptake of bosentan and a reduction of the induction effect on CYP2C9. Secondly, bosentan might induce CYP3A4 and CYP2C9 activity - acenocoumarol and warfarin mainly being metabolized by CYP2C9. This might be related to an increase in the elimination of the oral anticoagulant and the dramatic drop in the INR values. However, it did not seem to affect the effectiveness of lopinavir/ritonavir and dose adjustment of this might not be required. ${ }^{1}$ Other factors, such as genetic polymorphism in CYP2C9 (or in CYP2C29, a murine homologue of human CYP2C9), ${ }^{11}$ could represent another potential complicating factor in this interaction and should be studied in these patients. So, the genetic contribution to variation in dose requirements seems to be more pronounced for warfarin than acenocoumarol. ${ }^{8}$

Patients treated with bosentan, oral anticoagulants may need a significant increase in the anticoagulant dose to achieve an INR in the therapeutic range. However, randomised controlled trials comparing the dosage and safety of bosentan and oral anticoagulants in HIV patients are needed. Until then, the use of bosentan and oral anticoagulants in these patients not only seems to require closer monitoring during the first weeks of treatment with bosentan, or after an increase in its dose, but for even longer periods of time.

\section{References}

1. Dingemanse J, van Giersbergen PLM, Patat A, Nilsson PN. Mutual pharmacokinetic interactions between bosentan and lopinavir/ritonavir in healthy participants. Antiviral Therapy 2010;15:157-63.

2. Fuster V, Steele PM, Edwards WD, et al. Primary pulmonary hypertension: natural history and the importance of thrombosis. Circulation 1984;70:580-7.

3. Spangler ML, Saxena S. Warfarin and bosentan interaction in a patient with pulmonary hypertension secondary to bilateral pulmonary emboli. Clin Ther 2010;32: 53-6.

4. Murphey LM, Hood EH. Bosentan and warfarin interaction. Ann Pharmacother 2003; 37:1028-31.

5. Llibre JM, Romeu J, López E, Sirera G. Severe Interaction Between Ritonavir and Acenocoumarol. Ann Pharmacother 2002; 36:621-3.

6. Horn JR, Hansten PD, Chan L-N. Proposal of a new tool to evaluate drug interaction cases. Ann Pharmacother 2007;41:674-80.

7. Dingemanse J, van Giersbergen PL. Clinical pharmacology of bosentan, a dual endothelin receptor antagonist. Clin Pharmacokinetic 2004;43:1089-115.

8. Rane A, Lindh JD. Pharmacogenetics of Anticoagulants. Hum Genomics Proteomics 2010;2010:754919.

9. Yeh RF, Gaver VE, Patterson KB, et al. Lopinavir/ritonavir induces the hepatic activity of cytochrome P450 enzymes CYP2C9, CYP2C19, and CYP1A2 but inhibits the hepatic and intestinal activity of CYP3A as measured by a phenotyping drug cocktail in healthy volunteers. J. Acquir Immune Defic Syndr 2006;42:52-60.

10. Hartman JC, Brouwer K, Mandagere A, et al. Evaluation of the endothelin receptor antagonists, ambrisentan, darusentan, bosentan, and sitasentan as substrates and inhibitors of hepatobiliary transporters in sandwich-cultured human hepatocytes. Can J Physiol Pharmacol 2010;88: 682-91.

11. Guo Y, Weller P, Farrell E, et al. In Silico Pharmacogenetics: Warfarin Metabolism. Nat Biotechnol 2006;24:531-6. 